\title{
Generalized Fractional-Order Bernoulli Functions via Riemann-Liouville Operator and Their Applications in the Evaluation of Dirichlet Series
}

\author{
Jorge Sanchez-Ortiz $\mathbb{D}$ \\ Facultad de Matemáticas, Universidad Autónoma de Guerrero, Av. Lázaro Cárdenas S/N, Cd. Universitaria, Chilpancingo, \\ Guerrero, México, CP 39087, Mexico
}

Correspondence should be addressed to Jorge Sanchez-Ortiz; jsanchezmate@gmail.com

Received 5 September 2018; Accepted 29 November 2018; Published 12 December 2018

Academic Editor: Khalil Ezzinbi

Copyright (c) 2018 Jorge Sanchez-Ortiz. This is an open access article distributed under the Creative Commons Attribution License, which permits unrestricted use, distribution, and reproduction in any medium, provided the original work is properly cited.

In this work, we define a new class of functions of the Bernoulli type using the Riemann-Liouville fractional integral operator and derive a generating function for these class generalized functions. Then, these functions are employed to derive formulas for certain Dirichlet series.

\section{Introduction}

The Bernoulli polynomials are defined by the generating function [1]

$$
\sum_{n=0}^{\infty} B_{n}(x) \frac{t^{n}}{n !}=\frac{t e^{x t}}{e^{t}-1} \quad(|t|<2 \pi) .
$$

When $x=0, B_{n}=B_{n}(0)$ are called Bernoulli numbers. The following property is well known:

$$
B_{n}(x)=\sum_{j=0}^{n}\left(\begin{array}{l}
n \\
j
\end{array}\right) B_{n-j} x^{j} .
$$

Also, the Bernoulli polynomials are defined by the following Fourier series [2]:

$$
B_{n}(x)=-\frac{n !}{(2 \pi i)^{n}} \sum_{k=-\infty}^{\infty} k^{-n} e^{2 \pi i k x} \quad(0 \leq x<1) .
$$

Various generalizations of the Bernoulli polynomials have been proposed. For example, Natalini [3] gave the following generalization:

$$
E_{1, m+1}(t) e^{x t}=\sum_{n=0}^{\infty} B_{n}^{[m-1]}(x) \frac{t^{n}}{n !}
$$

where $E_{\alpha, \beta}(t)$ is the two-parametric Mittag-Leffler function, so that, obviously, $B_{n}(x):=B_{n}^{[0]}(x)$. Another generalization is given by Balanzario [4]:

$$
\mathscr{B}_{n}(x)=\int_{0}^{x} \mathscr{B}_{n-1}(y) d y+\int_{0}^{1}(y-1) \mathscr{B}_{n-1}(y) d y,
$$

where $\mathscr{B}_{0}(x)$ is given and $n \geq 1$. In case $\mathscr{B}_{0}(x)=1$ for $x \in$ $[0,1)$, then $\mathscr{B}_{n}(x) \cdot n$ ! is the usual $n$-th Bernoulli polynomial. Balanzario and Sanchez [5] derive the following generating function for $\mathscr{B}_{n}(x)$ defined in (5):

$$
\begin{aligned}
\sum_{k=0}^{\infty} \mathscr{B}_{k}(x) t^{k}= & \frac{t e^{x t}}{e^{t}-1}\left[a_{0}-\int_{0}^{1} \mathscr{B}_{0}^{\prime}(1-y) \frac{e^{t y}-1}{t} d y\right] \\
& +\int_{0}^{x} e^{t(x-y)} \mathscr{B}_{0}^{\prime}(y) d y,
\end{aligned}
$$

where $\mathscr{B}_{0}(x)$ is given and $a_{0}=\int_{0}^{1} \mathscr{B}_{0}(x) d x$; they used these generalized Bernoulli polynomials to derive formulas of certain Dirichlet series.

Rahimkhani et al. [6] define the fractional-order Bernoulli functions, such as the functions obtained by changing the variable $t$ to $x^{\alpha}$ in (3), and applied these functions for solving the fractional Fredholem-Volterra integrodifferential equations. 
In the present paper, new functions called generalized fractional-order Bernoulli functions are defined by a generalization of (5) and obtain a generalization of the generating function (6). Also, given a generalization of the Fourier series (3), we use these functions to derive formulas for certain Dirichlet series and finally, some examples are shown.

\section{Preliminaries}

In this section, we give some basic definitions and properties of fractional calculus theory which are used in this work.

Definition 1. The Riemann-Liouville fractional integral of order $\alpha \in \mathbb{R}^{+}$is defined by

$$
\left(I^{\alpha} f\right)(x)=\frac{1}{\Gamma(\alpha)} \int_{0}^{x} \frac{f(t)}{(x-t)^{1-\alpha}} d t,
$$

where $x>0$ and $\Gamma$ is the Gamma function.

It can be directly verified that

$$
\left(I^{\alpha} t^{\beta}\right)(x)=\frac{\Gamma(\beta)}{\Gamma(\beta+\alpha)} x^{\beta+\alpha-1},
$$

where $\alpha>0$ and $\beta>0$.

Definition 2. The Caputo fractional derivative of order $\alpha \epsilon$ $\mathbb{R}^{+}$is defined by

$$
\left(D^{\alpha} f\right)(x)=\left(I^{r-\alpha} D^{r} f\right)(x),
$$

where $D=d / d x, r=[\alpha]+1$ for $\alpha \notin \mathbb{N}_{0}$ and $r=\alpha$ for $\alpha \in \mathbb{N}_{0}$.

Now, when $\alpha \in \mathbb{R}^{+}$, the Caputo fractional differential operator $D^{\alpha}$ provides operation inverse to the RiemannLiouville fractional integration operator $I^{\alpha}$; the proof can be seen in [7].

Lemma 3. Let $\alpha \in \mathbb{R}^{+}$and $f(x)$ a continuous function in the interval $[0,1]$. Then, $\left(D^{\alpha} I^{\alpha} f\right)(x)=f(x)$.

Now, we define the Laplace transform of a function $f(x)$ of a variable $x \in \mathbb{R}^{+}$by

$$
\mathscr{L}[f(x)](k)=\int_{0}^{\infty} e^{-k x} f(x) d x \quad(k \in \mathbb{C}),
$$

if the integral converges and its inverse by

$$
\mathscr{L}^{-1}[f(k)](x)=\frac{1}{2 \pi i} \int_{\gamma-i \infty}^{\gamma+i \infty} e^{k x} f(k) d k,
$$

with $\gamma>\sigma$, where $\sigma$ is the abscissa of convergence.

Under suitable conditions, the Laplace transform of the Caputo fractional derivative $D^{\alpha} f$ is given by [7]

$$
\begin{aligned}
\mathscr{L}\left[D^{\alpha} f(x)\right](k)= & k^{\alpha} \mathscr{L}[f(x)](k) \\
& -\sum_{j=0}^{r-1} k^{\alpha-j-1}\left(D^{j} f\right)(0) .
\end{aligned}
$$

Definition 4. The two-parametric Mittag-Leffler function

$$
E_{\alpha, \beta}(z)=\sum_{k=0}^{\infty} \frac{z^{k}}{\Gamma(\alpha k+\beta)} \quad(\alpha>0, \beta \in \mathbb{C}),
$$

generalizes the classical Mittag-Leffler function

$$
E_{\alpha}(z)=\sum_{k=0}^{\infty} \frac{z^{k}}{\Gamma(\alpha k+1)} \quad(\alpha>0) .
$$

Using Definition 4, we obtain the formulas

$$
\begin{aligned}
& E_{1, m}(z)=\frac{1}{z^{m-1}}\left(e^{z}-\sum_{k=0}^{m-2} \frac{z^{k}}{k !}\right), \\
& \mathfrak{R} e\left[x^{\beta-1} E_{1, \beta}(i \lambda x)\right]=x^{\beta-1} E_{2, \beta}\left(-(\lambda x)^{2}\right), \\
& \Im m\left[x^{\beta-1} E_{1, \beta}(i \lambda x)\right]=\lambda x^{\beta} E_{2,1+\beta}\left(-(\lambda x)^{2}\right),
\end{aligned}
$$

where $m \in \mathbb{N}, \beta>0$, and $\lambda \in \mathbb{R}$. From above equations, we have

$$
\begin{gathered}
x^{m} E_{2,1+m}\left(-\lambda^{2} x^{2}\right)=\frac{1}{\lambda^{m}}\left(\cos \left(\frac{m \pi}{2}-\lambda x\right)\right. \\
\left.-\sum_{k=0}^{m-1} \frac{(\lambda x)^{k}}{k !} \cos \left(\frac{m \pi}{2}-\frac{k \pi}{2}\right)\right) .
\end{gathered}
$$

The following differentiation formula is an immediate consequence of Definition 4

$$
\left(\frac{d}{d z}\right)^{m}\left[z^{\beta-1} E_{\alpha, \beta}\left(z^{\alpha}\right)\right]=z^{\beta-m-1} E_{\alpha, \beta-m}\left(z^{\alpha}\right)
$$

$(m \in \mathbb{N})$.

Using Definition 4 and term-by-term integration, we arrive at

$$
\begin{aligned}
& \frac{1}{\Gamma(\alpha)} \int_{0}^{z}(z-t)^{\mu-1} E_{\alpha, \beta}\left(\lambda t^{\alpha}\right) t^{\beta-1} d t \\
& \quad=z^{\mu+\beta-1} E_{\alpha, \beta+\mu}\left(\lambda z^{\alpha}\right),
\end{aligned}
$$

where $\mu>0$ and $\beta>0$. From (18) we obtain

$$
\begin{aligned}
& \left(I^{\alpha} t^{n \alpha} E_{2,1+n \alpha}\left(\lambda t^{2}\right)\right)(x)=x^{(n+1) \alpha} E_{2,1+(n+1) \alpha}\left(\lambda x^{2}\right), \\
& \left(I^{\alpha} t^{1+n \alpha} E_{2,2+n \alpha}\left(\lambda t^{2}\right)\right)(x) \\
& \quad=x^{1+(n+1) \alpha} E_{2,2+(n+1) \alpha}\left(\lambda x^{2}\right) .
\end{aligned}
$$

It follow from the well-known discrete orthogonality relation

$$
\sum_{h=0}^{m-1} e^{2 \pi i h k / m}= \begin{cases}m, & \text { if } k \equiv 0(\bmod m), \\ 0, & \text { if } k \neq 0(\bmod m)\end{cases}
$$


and formula (18) that

$$
\begin{gathered}
\left(I^{\alpha} \cosh [\sqrt{\lambda} t]\right)(x)=x^{\alpha} E_{2, \alpha+1}\left(\lambda x^{2}\right) \\
\left(I^{\alpha} \frac{\operatorname{senh}[\sqrt{\lambda} t]}{\sqrt{\lambda}}\right)(x)=x^{\alpha+1} E_{2, \alpha+2}\left(\lambda x^{2}\right) \quad(\alpha>0) .
\end{gathered}
$$

Now, we state an important relation between the Laplace transform and Mittag-Leffler function; the proof can be seen in [8].

Lemma 5. The following formula is true:

$$
\int_{0}^{\infty} e^{-k x} x^{\beta-1} E_{\alpha, \beta}\left(a x^{\alpha}\right) d x=\frac{k^{\alpha-\beta}}{k^{\alpha}-a},
$$

where $\alpha>0, \beta>0$, and $k>|a|^{1 / \alpha}$.

\section{Generalized Fractional-Order Bernoulli Functions}

In this section, first we define a new set of fractionalorder Bernoulli functions by means of the Riemann-Liouville fractional integration operator.

Definition 6. Let $\mathscr{B}_{0}^{\alpha}(x)$ be a periodic function of period 1 . We define the fractional-order Bernoulli functions by

$$
\mathscr{B}_{n}^{\alpha}(x)=\left(I^{\alpha} \mathscr{B}_{n-1}^{\alpha}\right)(x)-\frac{1}{\alpha \Gamma(\alpha)} \int_{0}^{1} \frac{\mathscr{B}_{n-1}^{\alpha}(t)}{(1-t)^{-\alpha}} d t,
$$

where $\alpha>0$ and $n \in \mathbb{N}$.

In the case, $\alpha=1$, then $\mathscr{B}_{n}^{1}(x)$ are the generalization of the Bernoulli polynomials defined in (5). For example, when $\mathscr{B}_{0}^{\alpha}(x)=1$ for $0 \leq x<1$, the first two fractional-order Bernoulli functions are

$$
\begin{aligned}
\mathscr{B}_{1}^{\alpha}(x)= & \frac{x^{\alpha}}{\Gamma(1+\alpha)}-\frac{1}{\Gamma(2+\alpha)}, \\
\mathscr{B}_{2}^{\alpha}(x)= & \frac{x^{2 \alpha}}{\Gamma(1+2 \alpha)}-\frac{x^{\alpha}}{\Gamma(1+\alpha) \Gamma(2+\alpha)} \\
& +\frac{1}{(\Gamma(2+\alpha))^{2}}-\frac{1}{\Gamma(2+2 \alpha)} .
\end{aligned}
$$
ties:

The functions defined (25) satisfy the following proper-

$$
\begin{aligned}
& \qquad \int_{0}^{1} \mathscr{B}_{n}^{\alpha}(x) d x=0 \\
& \text { and }\left(D^{\alpha} \mathscr{B}_{n}^{\alpha}\right)(x)=\mathscr{B}_{n-1}^{\alpha}(x) .
\end{aligned}
$$

These assertions are followed by integrating (25) and Lemma 3, given that $\left(D^{\alpha} c\right)(x)=0$, for $c \in \mathbb{R}$.

In the following theorem, we obtain a generating function for the fractional-order Bernoulli functions defined in (25).
Theorem 7. Let $\mathscr{B}_{0}^{\alpha}(x)$ be a periodic function of period 1 . Suppose that $\mathscr{B}_{0}^{\alpha}(x)$ has a continuous derivative in the open interval $(0,1)$. Let $A_{0}=\int_{0}^{1} \mathscr{B}_{0}^{\alpha}(x) d x$ and $\left\{\mathscr{B}_{n}^{\alpha}\right\}$ be the sequence defined by (25). Then for $\alpha>0$,

$$
\begin{aligned}
\sum_{n=0}^{\infty} \mathscr{B}_{n}^{\alpha}(x) t^{n} & \\
= & \frac{E_{\alpha}\left(t x^{\alpha}\right)}{E_{\alpha, 2}(t)}\left[A_{0}-\int_{0}^{1} y D \mathscr{B}_{0}^{\alpha}(1-y) E_{\alpha, 2}\left(t y^{\alpha}\right) d y\right] \\
& +\int_{0}^{x} D \mathscr{B}_{0}(y) E_{\alpha}\left(t(x-y)^{\alpha}\right) d y .
\end{aligned}
$$

Proof. We proceed formally as in [9, Problem 9.785]. Consider the following fractional differential equation:

$$
D^{\alpha} G(x, t)-t G(x, t)=D^{\alpha} \mathscr{B}_{0}^{\alpha}(x),
$$

for a $\mathscr{B}_{0}^{\alpha}(x)$ given function and

$$
G(x, t)=\sum_{n=0}^{\infty} \mathscr{B}_{n}^{\alpha}(x) t^{n} .
$$

Applying the Laplace transform to (29) and using (12), we obtain

$$
\begin{aligned}
\mathscr{L} & {[G(x, t)](k) } \\
= & \frac{k^{\alpha}}{k^{\alpha}-t} \mathscr{L}\left[\mathscr{B}_{0}^{\alpha}(x)\right](k) \\
& \quad+\frac{1}{k^{\alpha}-t} \sum_{j=0}^{m-1} k^{\alpha-j-1}\left[\left(D^{j} G\right)(0, t)-\left(D^{j} \mathscr{B}_{0}^{\alpha}\right)(0)\right],
\end{aligned}
$$

where $m=[\alpha]+1$. Then, using the inverse Laplace transform in above equation, we arrive to the equation

$$
\begin{aligned}
G(x, t)= & \mathscr{L}^{-1}\left[\frac{k^{\alpha}}{k^{\alpha}-t} \mathscr{L}\left[\mathscr{B}_{0}^{\alpha}(x)\right](k)\right](x) \\
& +\left(G(0, t)-\mathscr{B}_{0}^{\alpha}(0)\right) \mathscr{L}^{-1}\left[\frac{k^{\alpha-1}}{k^{\alpha}-t}\right](x) .
\end{aligned}
$$

Therefore, by Lemma (25) and given that $\mathscr{L}[\delta(x)](k)=1$, we get

$$
\begin{aligned}
G(x, t)= & \left(G(0, t)-\mathscr{B}_{0}^{\alpha}(0)\right) E_{\alpha}\left(t x^{\alpha}\right)+E_{\alpha}\left(t x^{\alpha}\right) \\
& *\left(D \delta(x) * \mathscr{B}_{0}^{\alpha}(x)\right) \\
= & G(0, t) E_{\alpha}\left(t x^{\alpha}\right) \\
& +\int_{0}^{x} E_{\alpha}\left(t(x-y)^{\alpha}\right) D \mathscr{B}_{0}^{\alpha}(y) d y,
\end{aligned}
$$

where $\delta(x)$ is the Dirac delta function, and

$$
(f * g)(x)=\int_{0}^{x} f(y) g(x-y) d y
$$


is the convolution of the functions $f$ and $g$. Now, we integrate (33) from 0 to 1 with respect to $x$ and by (27) and (18) we obtain

$$
\begin{aligned}
A_{0}= & G(0, t) E_{\alpha, 2}(t) \\
& +\int_{0}^{1} \int_{0}^{x} E_{\alpha}\left(t(x-y)^{\alpha}\right) D \mathscr{B}_{0}^{\alpha}(y) d y d x .
\end{aligned}
$$

Solving for $G(0, t)$ and substituting in (33) we obtain our result.

Observe that if we set $\alpha=1$ and $\mathscr{B}_{0}^{\alpha}(x)=1$ for $x \in[0,1)$ in Theorem 7 , then we obtain the corresponding unification and generalization of the generating function (1) of the usual Bernoulli polynomials. In case $\alpha=1$ in Theorem 7, we obtain the generating function (6).

In the next theorem, we compute the fractional-order Bernoulli functions defined in (25) through the twoparametric Mittag-Leffler function.

Theorem 8. Let $\mathscr{B}_{0}^{\alpha}(x)$ be a periodic function of period one and piecewise continuous in the open interval $(0,1)$. Let $A_{0}$ and $\left\{\mathscr{B}_{n}^{\alpha}\right\}$ be as in Theorem 7. Then for $n \geq 1$,

$$
\begin{aligned}
& \mathscr{B}_{n}^{\alpha}(x)=A_{0}\left(\frac{x^{n \alpha}}{\Gamma(1+n \alpha)}-\frac{1}{\Gamma(2+n \alpha)}\right)+\sum_{k=1}^{n-1} \mathscr{B}_{k}^{\alpha}(0) \\
& \cdot\left(\frac{x^{(n-k) \alpha}}{\Gamma(1+(n-k) \alpha)}-\frac{1}{\Gamma(2+(n-k) \alpha)}\right) \\
& +2 \sum_{j=1}^{\infty} A_{j}\left(x^{n \alpha} E_{2,1+n \alpha}\left(-\lambda_{j}^{2} x^{2}\right)-E_{2,2+n \alpha}\left(-\lambda_{j}^{2}\right)\right) \\
& +4 \pi \sum_{j=1}^{\infty} j B_{j}\left(x^{1+n \alpha} E_{2,2+n \alpha}\left(-\lambda_{j}^{2} x^{2}\right)\right. \\
& \left.-E_{2,3+n \alpha}\left(-\lambda_{j}^{2}\right)\right)
\end{aligned}
$$

where $\lambda_{j}=2 \pi j$ and $A_{j}$ and $B_{j}$ are the Fourier coefficients of $\mathscr{B}_{0}^{\alpha}(x)$.

Proof. The proof is by mathematical induction on $n$. Since $B_{0}^{\alpha}(x)$ is piecewise continuous, then we can consider its Fourier series

$$
B_{0}^{\alpha}(x)=A_{0}+2 \sum_{j=1}^{\infty}\left(A_{j} \cos \left(\lambda_{j} x\right)+B_{j} \sin \left(\lambda_{j} x\right)\right) .
$$

Let $n=1$. Then by (22), (23), and (25) we obtain

$$
\begin{gathered}
\mathscr{B}_{1}^{\alpha}(x)=\left(I^{\alpha} A_{0}\right)(x)+2 \sum_{j=1}^{\infty}\left(A_{j}\left(I^{\alpha} \cos \left(\lambda_{j} t\right)\right)(x)\right. \\
\left.+B_{j}\left(I^{\alpha} \sin \left(\lambda_{j} t\right)\right)(x)\right)-\frac{A_{0}}{\alpha \Gamma(\alpha)} \int_{0}^{1} \frac{1}{(1-t)^{-\alpha}} d t \\
+\frac{2}{\alpha \Gamma(\alpha)} \sum_{j=1}^{\infty} \int_{0}^{1} \frac{A_{j} \cos \left(\lambda_{j} t\right)+B_{j} \sin \left(\lambda_{j} t\right)}{(1-t)^{-\alpha}} d t
\end{gathered}
$$

$$
\begin{aligned}
& =A_{0}\left(\frac{x^{\alpha}}{\Gamma(1+\alpha)}-\frac{1}{\Gamma(2+\alpha)}\right) \\
& +2 \sum_{j=1}^{\infty} A_{j}\left(x^{\alpha} E_{2,1+\alpha}\left(-\lambda_{j}^{2} x^{2}\right)-E_{2,2+\alpha}\left(-\lambda_{j}^{2}\right)\right) \\
& +2 \sum_{j=1}^{\infty} \lambda_{j} B_{j}\left(x^{1+\alpha} E_{2,2+\alpha}\left(-\lambda_{j}^{2} x^{2}\right)-E_{2,3+\alpha}\left(-\lambda_{j}^{2}\right)\right) .
\end{aligned}
$$

Now, we assume the theorem true for a given $n$ and we will prove that it is valid for $n+1$. From (25)

$$
\mathscr{B}_{n+1}^{\alpha}(x)=\left(I^{\alpha} \mathscr{B}_{n}^{\alpha}\right)(x)-\frac{1}{\alpha \Gamma(\alpha)} \int_{0}^{1} \frac{\mathscr{B}_{n}^{\alpha}(t)}{(1-t)^{-\alpha}} d t
$$

applying (8), (19), and (20) and the above equation we get the result.

\section{Evaluation by Certain Dirichlet Series}

For the proof of the following theorems one proceeds as in Balanzario [10], using Theorem 8 and (16).

Theorem 9. Let $\left\{f_{j}\right\}$ be a sequence of complex numbers of period $T$, so that $f_{j+T}=f_{j}$ for all $j \in \mathbb{N}$. Let $\mathscr{B}_{0}^{\alpha}(x),\left\{\mathscr{B}_{n}^{\alpha}\right\}$,

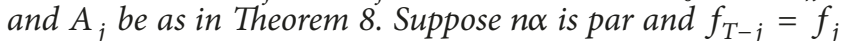
for each $j \in\{1,2, \ldots, T-1\}$ and suppose no is impar and $f_{T-j}=-f_{j}$ for each $j \in\{1,2, \ldots, T-1\}$ and $f_{T}=0$. Then

$$
\begin{aligned}
\sum_{j=1}^{\infty} \frac{A_{j}}{j^{n \alpha}}\left(f_{j}-\sum_{k=0}^{n \alpha-1} \gamma_{k}^{n \alpha} \frac{(2 \pi j)^{k}}{k !} \cos \left(\frac{\pi}{2}(n \alpha-k)\right)\right) \\
=\frac{(2 \pi)^{n \alpha}}{2}\left(\sum_{m=1}^{T} \beta_{m}^{n \alpha} \mathscr{B}_{n}^{\alpha}\left(\frac{m}{T}\right)\right. \\
-\frac{A_{0}}{T^{n \alpha}} \sum_{m=1}^{T} \beta_{m}^{n \alpha} \frac{m^{n \alpha}}{\Gamma(1+n \alpha)} \\
\left.-\sum_{k=1}^{n-1} \frac{\gamma_{(n-k) \alpha}^{n \alpha}}{\Gamma(1+(n-k) \alpha)} \mathscr{B}_{k}^{\alpha}(0)\right),
\end{aligned}
$$

where

$$
\begin{aligned}
\beta_{m}^{n \alpha} & =\frac{1}{T} \sum_{k=1}^{T} f_{k} \cos \left(\frac{n \alpha \pi}{2}-2 \pi m \frac{k}{T}\right), \\
\gamma_{k}^{n \alpha} & =\frac{1}{T^{k}} \sum_{m=1}^{T} m^{k} \beta_{m}^{n \alpha} .
\end{aligned}
$$

Theorem 10. Assume the notation of Theorem 10. If $n \alpha$ is impar and $f_{T-j}=f_{j}$ for each $j \in\{1,2, \ldots, T-1\}$ and if $n \alpha$ is 
par and $f_{T-j}=-f_{j}$ for each $j \in\{1,2, \ldots, T-1\}$ and $f_{T}=0$, then

$$
\begin{aligned}
\sum_{j=1}^{\infty} & \frac{B_{j}}{j^{\alpha n}}\left(-f_{j}+\sum_{k=0}^{n \alpha} \varphi_{k}^{n \alpha} \frac{(2 \pi j)^{k}}{k !} \sin \left(\frac{\pi}{2}(n \alpha-k)\right)\right) \\
& =\frac{(2 \pi)^{n \alpha}}{2}\left(\sum_{m=1}^{T} \mu_{m}^{n \alpha} \mathscr{B}_{n}^{\alpha}\left(\frac{m}{T}\right)\right. \\
& -\frac{A_{0}}{T^{n \alpha}} \sum_{m=1}^{T} \mu_{m}^{n \alpha} \frac{m^{n \alpha}}{\Gamma(1+n \alpha)} \\
- & \left.\sum_{k=1}^{n-1} \frac{\varphi_{(n-k) \alpha}^{n \alpha}}{\Gamma(1+(n-k) \alpha)} \mathscr{B}_{k}^{\alpha}(0)\right)
\end{aligned}
$$

where

$$
\begin{aligned}
\mu_{m}^{n \alpha} & =\frac{1}{T} \sum_{k=1}^{T} f_{k} \sin \left(\frac{n \alpha \pi}{2}-2 \pi m \frac{k}{T}\right), \\
\varphi_{k}^{n \alpha} & =\frac{1}{T^{k}} \sum_{m=1}^{T} m^{k} \mu_{m}^{n \alpha} .
\end{aligned}
$$

Finally, some examples are given.

Example 1. As the first example, we consider $\mathscr{B}_{0}^{\alpha}(x)$ be of period one such that $\mathscr{B}_{0}^{\alpha}(x)=x^{1 / 2}$ for $0 \leq x<1$. Let $\alpha=1 / 2, n=2, T=8, \lambda_{1}=\lambda_{5}=-1, \lambda_{3}=\lambda_{7}=1$, and $\lambda_{2}=\lambda_{4}=\lambda_{6}=\lambda_{8}=0$. Applying Theorem 9, we get

$$
\sum_{j=1}^{\infty} \frac{F_{s}(2 \sqrt{j})}{j^{5 / 2}}=\frac{\pi^{2}}{6}(5-3 \sqrt{3})
$$

where $F_{s}(z)$ is the Fresnel sine integral given by $\int_{0}^{z} \sin \left(\pi t^{2} / 2\right) d t$.

Example 2. Here is another example of Theorem 9. Let $\mathscr{B}_{0}^{\alpha}(x)$ be of period one such that $\mathscr{B}_{0}^{\alpha}(x)=x \cos (\pi x)$ for $0 \leq x<1$. Let $\alpha=2, n=1, T=10, \lambda_{1}=\lambda_{2}=\lambda_{3}=\lambda_{4}=\lambda_{6}=\lambda_{7}=$ $\lambda_{8}=\lambda_{9}=1$, and $\lambda_{5}=\lambda_{10}=0$. Then

$$
\begin{aligned}
\sum_{j=1}^{\infty} \frac{\left(1+4 j^{2}\right)}{j^{2}\left(1-4 j^{2}\right)^{2}} \lambda_{j} \\
=-\frac{\pi}{5}(\sqrt{2(5-\sqrt{5})}+\sqrt{2(5+\sqrt{5})}) \\
\quad+\frac{\pi^{2}}{50}(27-2 \sqrt{5}) .
\end{aligned}
$$

Example 3. Let $\mathscr{B}_{0}^{\alpha}(x)$ be of period one such that $\mathscr{B}_{0}^{\alpha}(x)=$ $x^{1 / 2} \sin (\pi x)$ for $0 \leq x<1$. Let $\alpha=1 / 2, n=2, T=8$, $\lambda_{1}=\lambda_{3}=\lambda_{5}=\lambda_{7}=1$, and $\lambda_{2}=\lambda_{4}=\lambda_{8}=0$. By applying Theorem 10, we obtain

$$
\begin{aligned}
& \sum_{j=1}^{\infty}\left(\frac{F s(\sqrt{4 j+2})}{(2 j+1)^{3 / 2}}-\frac{F_{s}(\sqrt{4 j-2})}{(2 j-1)^{3 / 2}}\right) \lambda_{j}=\frac{\pi}{2}(\sqrt{2} \\
& \left.\quad+F_{c}(\sqrt{2})\right)+\frac{\pi^{3}}{10}\left(-4 \sqrt{2}_{1} F_{2}\left(\frac{5}{4} ; \frac{3}{2}, \frac{9}{4} ;-\frac{\pi^{2}}{4}\right)\right. \\
& \left.\quad+{ }_{1} F_{2}\left(\frac{5}{4} ; \frac{3}{2}, \frac{9}{4} ;-\frac{\pi^{2}}{16}\right)\right),
\end{aligned}
$$

where $F_{c}(z)$ is the Fresnel cosine integral and ${ }_{1} F_{2}$ is the hypergeometric function defined by

$$
{ }_{1} F_{2}(a ; b, c ; z)=\sum_{k=0}^{\infty} \frac{(a)_{k}}{(b)_{k}(c)_{k}} \frac{z^{k}}{k !}, \quad(a)_{k}=\frac{\Gamma(a+k)}{\Gamma(a)} .
$$

\section{Data Availability}

No data were used to support this study.

\section{Conflicts of Interest}

The author declares that there are no conflicts of interest regarding the publication of this paper.

\section{References}

[1] L. Euler, "Methodus generalis summandi progressiones," Commentarii Academiae Scientiarum Petropolitanae, vol. 6, pp. 6897, 1738.

[2] D. H. Lehmer, "A new approach to Bernoulli polynomials," The American Mathematical Monthly, vol. 95, no. 10, pp. 905-911, 1988.

[3] P. Natalini and A. Bernardini, "A generalization of the bernoulli polynomials," Journal of Applied Mathematics, vol. 3, pp. 155$163,2003$.

[4] E. P. Balanzario, "A generalized Euler-Maclaurin formula for the Hurwitz zeta function," Mathematica Slovaca, vol. 56, no. 3, pp. 307-316, 2006

[5] E. P. Balanzario and J. Sanchez-Ortiz, "A generating function for a class of generalized Bernoulli polynomials," Ramanujan Journal, vol. 19, no. 1, pp. 9-18, 2009.

[6] P. Rahimkhani, Y. Ordokhani, and E. Babolian, "Fractionalorder Bernoulli functions and their applications in solving fractional Fredholem-Volterra integro-differential equations," Applied Numerical Mathematics, vol. 122, pp. 66-81, 2017.

[7] A. A. Kilbas, H. M. Srivastava, and J. J. Trujillo, Theory and Applications of Fractional Differential Equations, Elsevier, San Diego, Calif, USA, 2006.

[8] R. Gorenflo, A. A. Kilbas, F. Mainardi, and S. V. Rogosin, MittagLeffler Functions, Related Topics and Applications, Springer, Berlin, Germany, 2014.

[9] R. P. Agnew, Differential Equations, McGraw-Hill, New York, NY, USA, 2nd edition, 1960.

[10] E. P. Balanzario, "Evaluation of Dirichlet series," The American Mathematical Monthly, vol. 108, no. 10, pp. 969-971, 2001. 


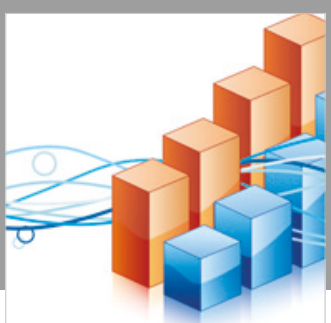

Advances in

Operations Research

\section{-n-m}
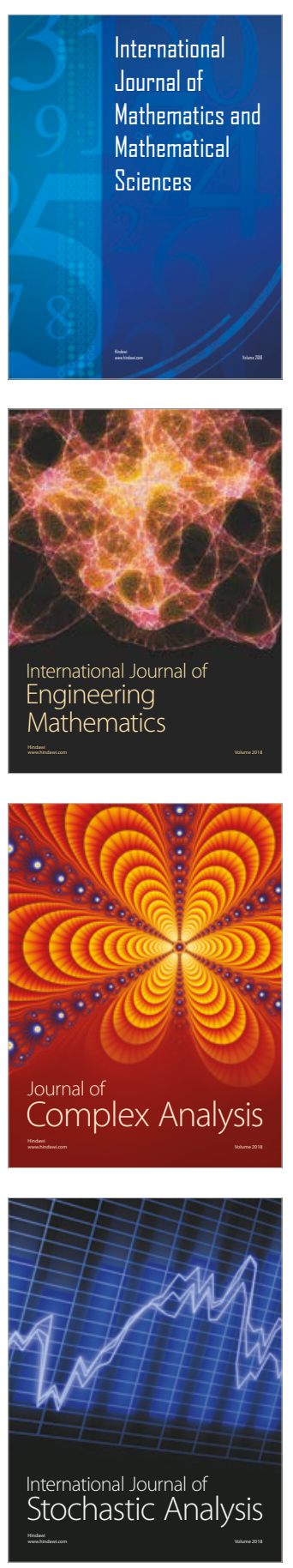
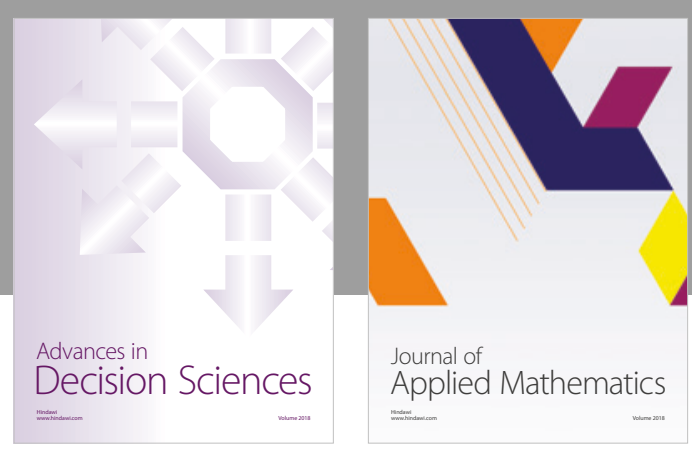

Journal of

Applied Mathematics
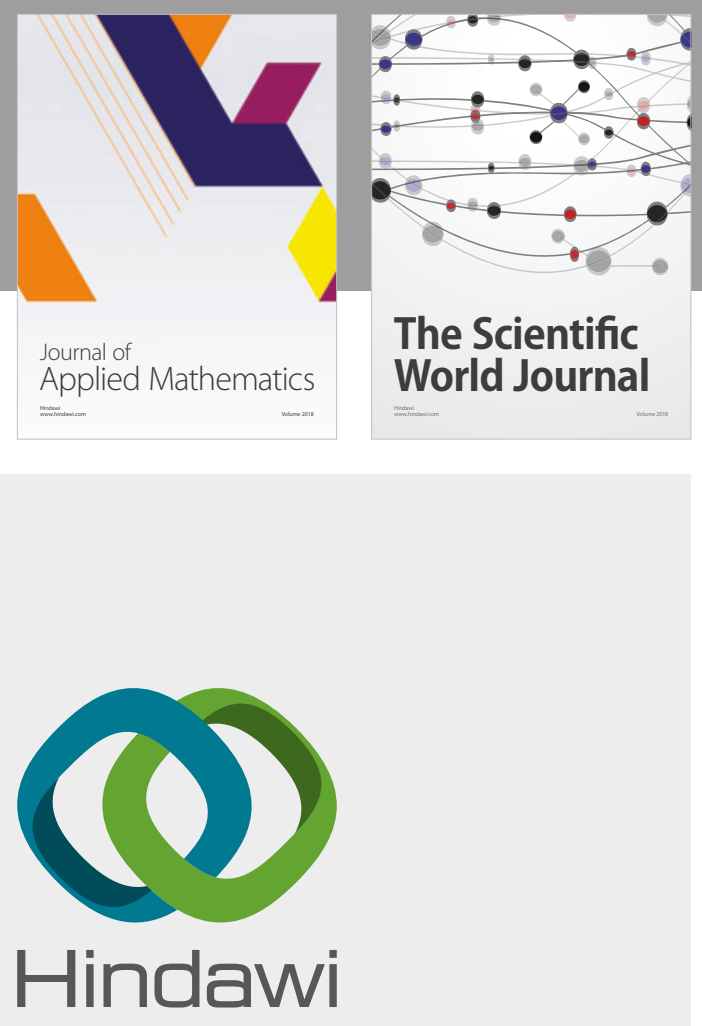

Submit your manuscripts at

www.hindawi.com

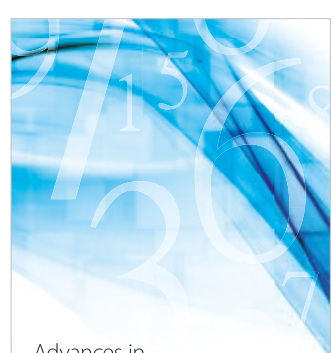

Advances in
Numerical Analysis
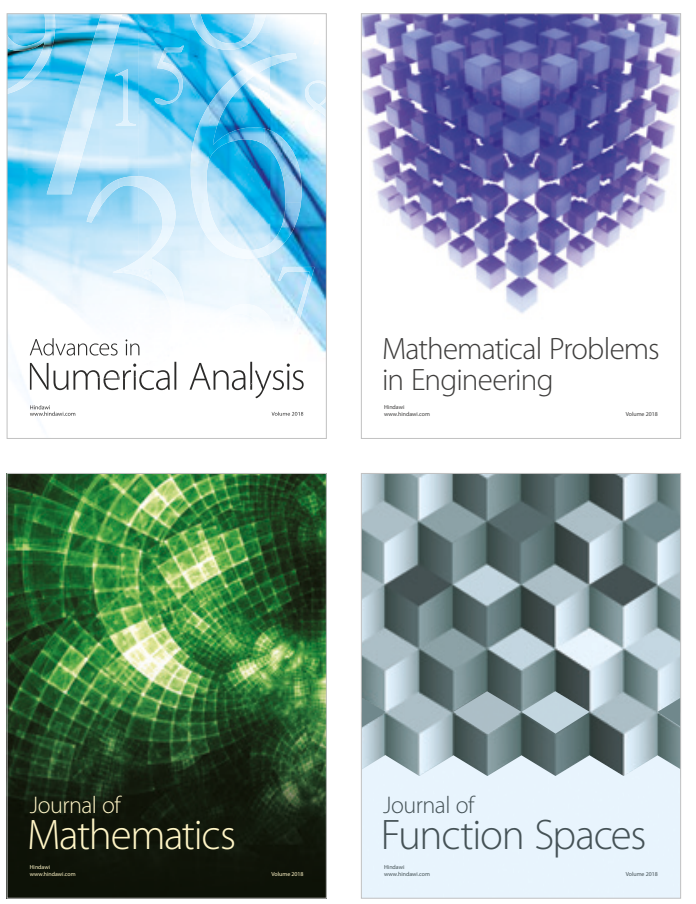

Mathematical Problems in Engineering

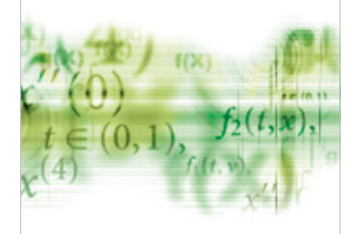

International Journal of

Differential Equations

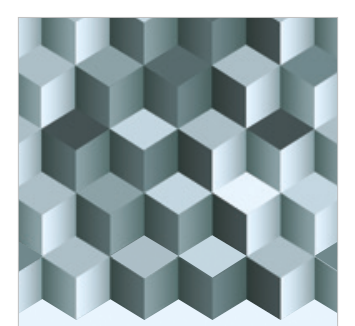

Journal of

Function Spaces

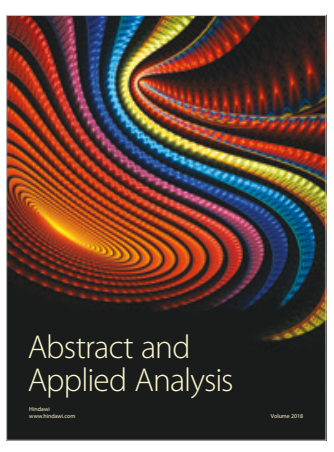

The Scientific

World Journal

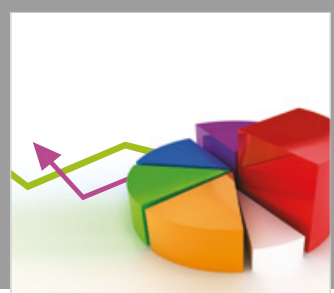

Journal of

Probability and Statistics
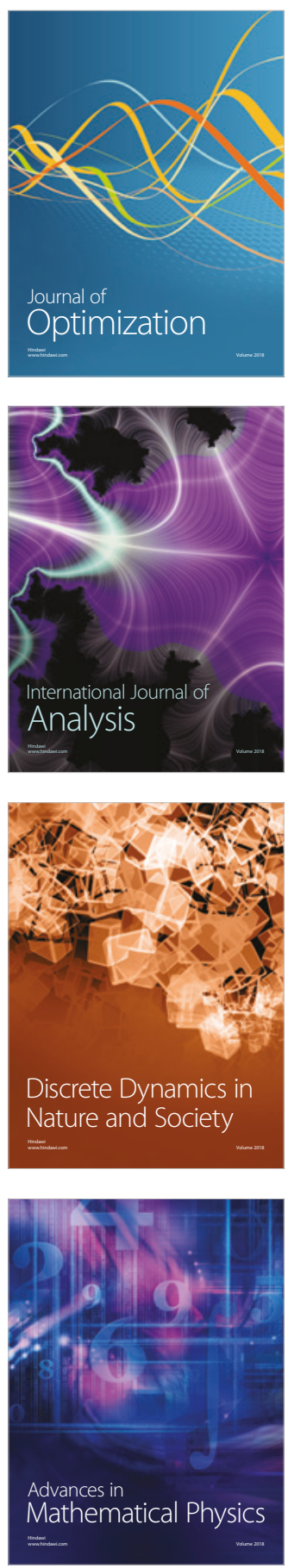